\title{
Factors Influencing Discussion of Cancer Genetic Testing with Health-Care Providers in a Population-Based Survey
}

\author{
Sukh Makhnoon ${ }^{a}$ Robert Yu ${ }^{b}$ Sonia A. Cunningham ${ }^{c}$ Susan K. Peterson ${ }^{a}$ \\ Sanjay Shete ${ }^{b, c, d}$ \\ aDepartment of Behavioral Science, UT MD Anderson Cancer Center, Houston, TX, USA; bepartment of Biostatistics, \\ UT MD Anderson Cancer Center, Houston, TX, USA; 'Department of Epidemiology, UT MD Anderson Cancer Center, \\ Houston, TX, USA; dDivision of Cancer Prevention and Population Sciences, UT MD Anderson Cancer Center, \\ Houston, TX, USA
}

\section{Keywords}

Cancer $\cdot$ Genetic testing $\cdot$ Healthcare providers $\cdot$ Discussion

\begin{abstract}
Introduction: Discussion of cancer genetic testing with health-care providers (HCPs) is necessary to undergo testing to inform cancer risk assessment and prevention. Given the rapid evolution in genetic testing practice in oncology, we describe the current landscape of population-level cancer genetic testing behaviors. Methods: A questionnaire including items regarding discussion of cancer genetic testing with HCPs was administered to a nonprobability sample ( $N=$ 2,029 ) of the Texas population. Results: Overall, $11 \%$ of respondents discussed cancer genetic testing with HCPs. In multivariable analysis, discussion was significantly related to having a personal history of breast/ovarian/colon cancer (OR $=11.57,95 \% \mathrm{Cl}=5.34-25.03)$, personal history of other cancer (OR $=3.18,95 \% \mathrm{Cl}=1.69-5.97)$, and health information-seeking behaviors ( $O R=1.73,95 \% \mathrm{Cl}=1.12-2.66)$. Surprisingly, respondents who believed that inherited predispositions in addition to other modifiable risk factors cause cancer were less likely to discuss genetic testing compared to those who did not believe that inherited cancer predispo-
\end{abstract}

karger@karger.com

(c) 2021 S. Karger AG, Basel

www.karger.com/phg

Karger! sitions cause cancer ( $\mathrm{OR}=0.54,95 \% \mathrm{Cl}=0.36-0.79)$. Discussion: The high discussion rate may be attributed to increased public awareness of genetic testing and adoption of more inclusive clinical genetic testing guidelines. The findings suggest that efforts to increase public awareness of the utility of genetic testing on personalized cancer risk assessment and cancer prevention are needed.

(c) 2021 S. Karger AG, Basel

\section{Introduction}

Up to $10 \%$ of all cancers have been attributed to inherited gene mutations [1] and clinical genetic testing can identify mutation carriers and estimate their lifetime cancer risks. Health-care providers (HCPs) play a critical role in identifying mutation carriers through genetic testing [2]. They act as gatekeepers to most clinical genetic testing, including that for cancer predisposition, which requires some form of physician authorization. Even direct-to-consumer (DTC) genetic test results, which do not require physician involvement, must be clinically confirmed before they can be used to make health-care decisions. As such, discussion of genetic testing options
Correspondence to:

Sanjay Shete, sshete@mdanderson.org 
with HCPs usually serves as the first step toward perusal of clinical genetic testing. With increased popularity, availability, and use of new genetic-based testing strategies, the general public is becoming more aware and interested in genetic testing services [3]. At this early stage of test adoption, patients rely on their HCPs for guidance regarding whether and how to undergo such testing for cancer [4]. As such, HCPs are ideally positioned to assess health risks and apply evidence-based guidelines to determine whether referral to genetic services is warranted. HCPs are a trusted source of medical information for most patients [5] and play a critical role in ensuring that individuals who meet the eligibility criteria are recommended for genetic testing. Only limited information on cancer genetic testing behaviors at the population level is available, and given the rapid evolution of cancer genetics, more up-to-date data representing the current landscape are urgently needed.

Discussion of genetic testing with an HCP may be warranted for those with a personal or family history of heritable cancer who meet the National Comprehensive Cancer Network's criteria for eligibility $[6,7]$. We know that well-educated women are more likely to be aware of and proactively discuss genetic testing with their HCPs, especially testing for Hereditary Breast and Ovarian Cancer, as it is the most commonly discussed hereditary cancer in clinical genetics [8]. A population-level assessment conducted in 2015 by the National Health Interview Survey (NHIS) found that only $2.25 \%$ of the population discussed genetic testing with their HCP [9]. However, it is likely that public awareness and interest in genetic testing may have since changed. First, there is an insurance-mandated requirement for pretest genetic counseling in order to be reimbursed for testing $[10,11]$ which likely increases the demand for HCP services such as genetic counselors and geneticists. Second, as a result of recently enacted inclusive genetic referral guidelines, more people are eligible for genetic counseling and need providers' assistance to receive guideline-compliant genetic services. Discussion of genetic testing is a necessary part of routine clinical care, especially in oncology, where HCPs such as genetic counselors and oncologists are involved in counseling, ordering, evaluating, and disclosing results from genetic testing for cancer susceptibility. The present study aims to provide updated populationlevel data on discussion of genetic testing among adults to represent the rapidly evolving landscape of genetic testing. Investigations will extend to understanding the relationship between discussion of genetic testing and various psychosocial and sociodemographic variables (e.g., health information-seeking behaviors and cancer risk beliefs).

In general, this study was guided by the widely used Health Belief Model (HBM) [12] as a relevant theoretical framework for understanding the determinants of individual-level health behavior such as the discussion of genetic testing. HBM asserts that people will take action if they perceive: the illness is serious (perceived severity); perceived person risk for the illness (perceived susceptibility); actions taken to control the illness are effective (perceived benefits) relative to the impediments (perceived barriers). Exposure to factors that prompt action; belief that they can successfully perform the action to control the illness (self-efficacy); and psychosocial factors (e.g., worry) may also facilitate behavior change. HBM is well validated and has been shown to predict a broad variety of health behaviors including cancer prevention behaviors in genetics $[13,14]$. Additionally, we enhanced the HBM with constructs known to influence health decision-making, such as information seeking and related beliefs [15].

This study describes discussion of genetic testing for hereditary cancer risk among residents of the state of Texas. Texas is the second most populous US state and has rich racial and ethnic diversity with $41.2 \%$ of the population being non-Hispanic White, 38.7\% Hispanic, and 5.2\% Asian [16]. Specific estimates on current cancer genetic testing behaviors, cancer risk perceptions, and information-seeking behaviors among the racially and ethnically diverse Texas population can inform cancer prevention and control efforts. We sought to understand the perspectives and behaviors of cancer genetic testing and their sociodemographic predictors among $>2,000$ Texas residents.

\section{Materials and Methods}

Survey Design and Participants

The Texas Population Health Assessment survey is a nonprobability-based population survey of Texas residents and has been described in detail elsewhere [17]. Briefly, 15 NCI-designated cancer centers were funded to collect critical catchment-area data on population health behavior, cancer knowledge, access to care, cancer communication, risk perceptions, and attitudes. The Texas survey included 153 items in total, 42 if which were core questions that were common across cancer centers. The complete survey was primarily comprised of measures retrieved from NHIS [18], Health Information National Trends Survey (HINTS) [19], and Behavioral Risk Factor Surveillance System Questionnaire [20] instruments. The survey was translated to Mexican Spanish by Masterword Services, Inc., Houston, TX and administered to Texas residents through Qualtrics in both English and Spanish. Participants 
were recruited through the online research panels retained by Qualtrics. Sampling was targeted to match the demographic distribution of Texas and was stratified by sex, ethnicity, race, income, and rurality. For the purposes of data collection, rural-urban residence was defined by matching ZIP code to county designations $[12,13]$. The survey was conducted from February 5 to March 5, 2018. In total, 5,628 responders (including screeners for eligibility) initiated the survey, of which 1,600 dropped out. A total of 2,050 participants with complete responses were obtained. Participants were reimbursed with $\$ 10$ or its equivalent for their time. MD Anderson's Institutional Review Board approved the study protocol and survey instrument before launch and all survey respondents provided informed consent (PA16-0724).

\section{Outcome Measure}

Discussion of Genetic Testing

The primary outcome measure analyzed in this study is discussion of cancer genetic testing with an HCP. Participants were provided with a brief introduction to Genetic Testing: "The following question refers to genetic TESTING for cancer risk. That is, testing your blood to see if you carry genes which may predict a greater chance of developing cancer at some point in your life. This does NOT include tests to determine if you have cancer now. DO NOT include self-testing kits administered at home." Following the introduction, participants were asked, "Have you EVER DISCUSSED the possibility of getting a genetic test for cancer risk with a doctor or other health professional." Possible responses were Yes or No.

\section{Predictor Measures}

Perceived severity was measured using one item of the cancer risk perception scale as used in HINTS [19], "Compared to other people your age, how likely are you to get cancer in your lifetime?" with responses on a 5-point Likert scale of very unlikely [1] to very likely [5]. Personal and family history of cancer also informs perceived illness severity. Personal history of cancer was measured using a single yes/no item: "Have YOU ever been diagnosed as having cancer?" with a follow-up question about specific cancer types. Family history of cancer was measured using a single item: "Have any of YOUR BLOOD RELATIVES (immediate or extended) ever had cancer?" with options of yes, no, or not sure.

Perceived susceptibility was measured using a subscale of the cancer belief scale [17]: Which of the following do you believe has a significant effect on whether or not the average person develops cancer? Included among the 8 answer choices were overweight/ obesity, tobacco, and inherited predisposition such as cancer genes.

Perceived benefit of cancer genetic testing was measured by assessing participants' agreement with the following statements on a 4-point Likert scale, from strongly agree (1) to strongly disagree (4), as used by HINTS [19]: "there's not much you can do to lower your chances of getting cancer." The remaining subscales were used to measure perceived barriers including: "it seems like everything causes cancer," "there's not much you can do to lower your chances of getting cancer," "there are so many different recommendations about preventing cancer, it's hard to know which ones to follow," "when I think about cancer, I automatically think about death," and "cancer is most often caused by a person's behavior or lifestyle." This is a well-used measure shown to have predictive capability for cancer-preventive health behaviors [17].
Health information seeking was measured using one item: "Have you ever looked for information about health or medical topics from any source?" (yes or no), which is known to be positively associated with genetic information seeking $[21,22]$. Those who responded yes were asked about the source of information: "The most recent time you looked for information about health or medical topics, where did you go first?" With 14 possible options including books, cancer organization, family, friend/coworker, etc. [23]. Information seeking ability was assessed using 4 items that shared a common stem: "Based on your most recent search for information about health, how much do you agree or disagree with the following statements?" - It took a lot of effort to get the information you needed; you felt frustrated during your search for the information; you were concerned about the quality of information; and that the information you found was hard to understand. Each item was scored on a 4-point Likert scale from strongly agree (1) to strongly disagree (4) to indicate ease of information seeking. In addition to information seeking, trust in an information source as well as ability to seek information are known to have significant effects on health-care decisions and outcomes [24, 25]. Therefore, trust in information source was measured using 9 items that shared a question stem: "In general, how much would you trust information about health or medical topics from each of the following?" - a doctor, family or friends, newspapers or magazines, radio, internet, television, government health agencies, charitable organizations, religious organizations, and leaders. Each item was scored on a 4-point scale from not at all (1) to a lot (4). To assess cancer risk information avoidance, participants were asked to rate their agreement with the following statement: "I'd rather not know my chance of getting cancer," on a 4 point scale of strongly agree to strongly disagree, as adapted from HINTS $[19,26]$.

Sociodemographic characteristics including sex, age, ethnicity/race, education, marital status, total household income, household size, employment status, and residence were also assessed.

\section{Statistical Analysis}

Survey weights were calculated using a 3-dimensional raking approach using the post-stratification variables: 3 category age (18-44, 45-69, and 60 and over); sex; and 4-category race/ethnicity (non-Hispanic White, non-Hispanic Black, Hispanic, and other). All analyses were carried out using Statistical Analysis Software or SAS (version 9.4). Weighted prevalence estimates of sociodemographic characteristics and health-seeking behaviors were estimated for those who discussed and those who did not discuss genetic testing. The outcome variable of interest discussion of genetic testing was treated as a binary response variable. Age was treated as a continuous variable and all other predictors: sex; race/ ethnicity; educational attainment; marital status; household income; location of residence; health insurance status; personal and family history of cancer; health information-seeking behavior; cancer risk perception; and cancer belief and preferences were treated as categorical variables. Weighted multivariate logistic regressions with survey weights were carried out using PROC SURVEYLOGISTIC to model the likelihood of the association between discussion of genetic testing with an HCP with sociodemographic characteristics, health risk beliefs, and perceptions, as well as health-seeking behaviors. Statistical significance was determined at 2 -sided $p<0.05$. 
Table 1. Descriptive statistics $(N=2,029)$ of survey respondents stratified by discussion of genetic testing with an HCP

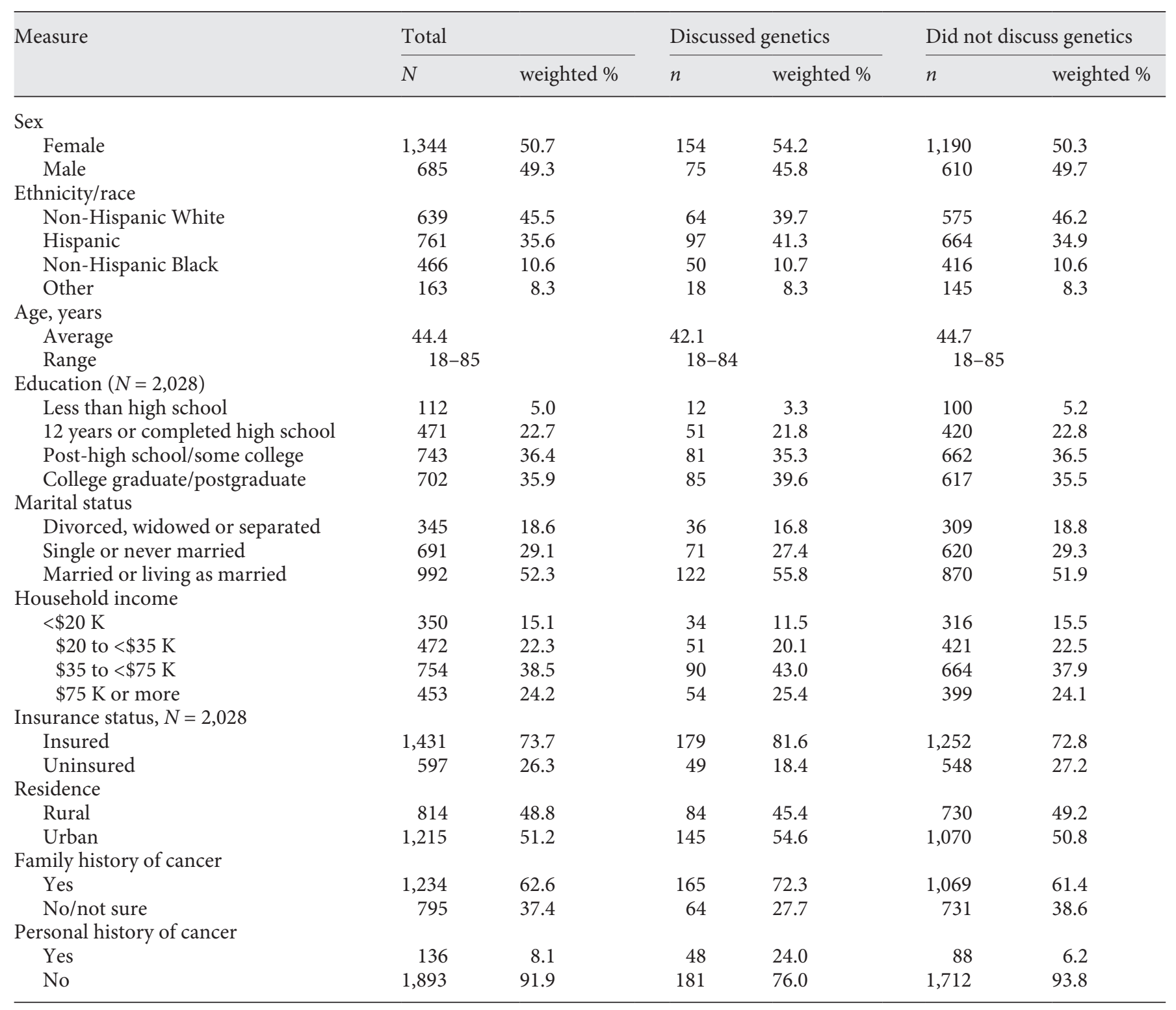

HCP, health-care provider.

\section{Results}

\section{Participant Characteristics}

Descriptive data and weighted percentages for the survey respondents are presented in Table 1 . The data set included 2,029 observations weighted to represent 25,538,321 noninstitutionalized civilian Texas residents (age 18 and older) with complete response for the study outcome. Of these, $50.7 \%$ were female, $45.5 \%$ (a majority) were non-Hispanic White, mean age was 44.4 years, and $35.9 \%$ had attained a college or postgraduate level of education. Most respondents had a household income of $\$ 35 \mathrm{~K}$ to $<\$ 75 \mathrm{~K}$ per year (38.5\%), lived in an urban area within Texas (51.2\%), and were married or living as married $(52.3 \%)$. A total of $91.9 \%$ did not have a personal history of cancer although $62.6 \%$ had family history of cancer. Personal history of the most common hereditary cancer conditions, i.e., breast, ovarian, or colorectal cancers were reported by $2.1 \%(n=42)$ of the study respondents. 


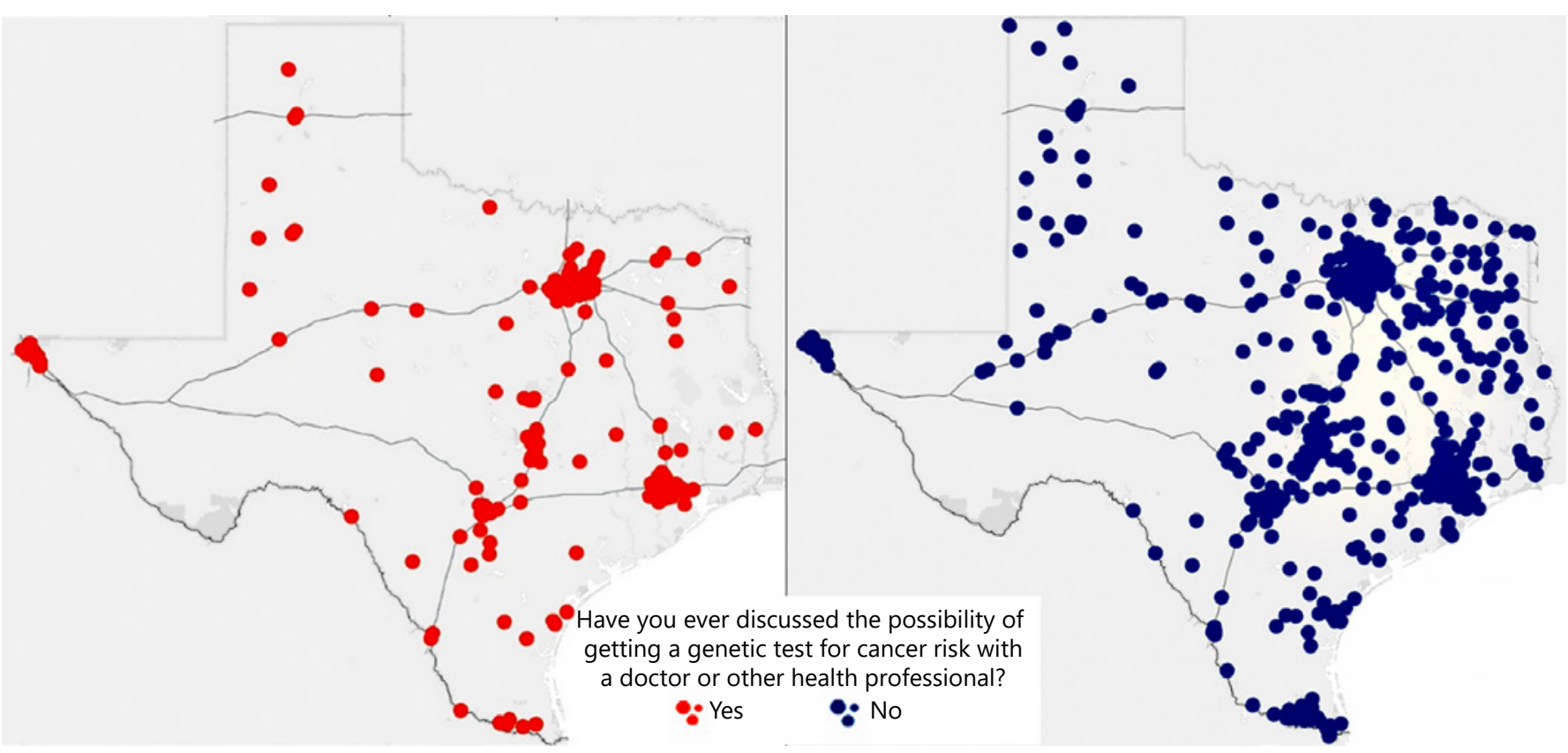

Fig. 1. Distribution of cancer genetic test discussions among individuals in Texas. Geocoded locations of survey respondents who discussed genetic testing with a provider in red $(n=229)$ and respondents who did not discuss genetic testing in blue $(n=1,800)$.

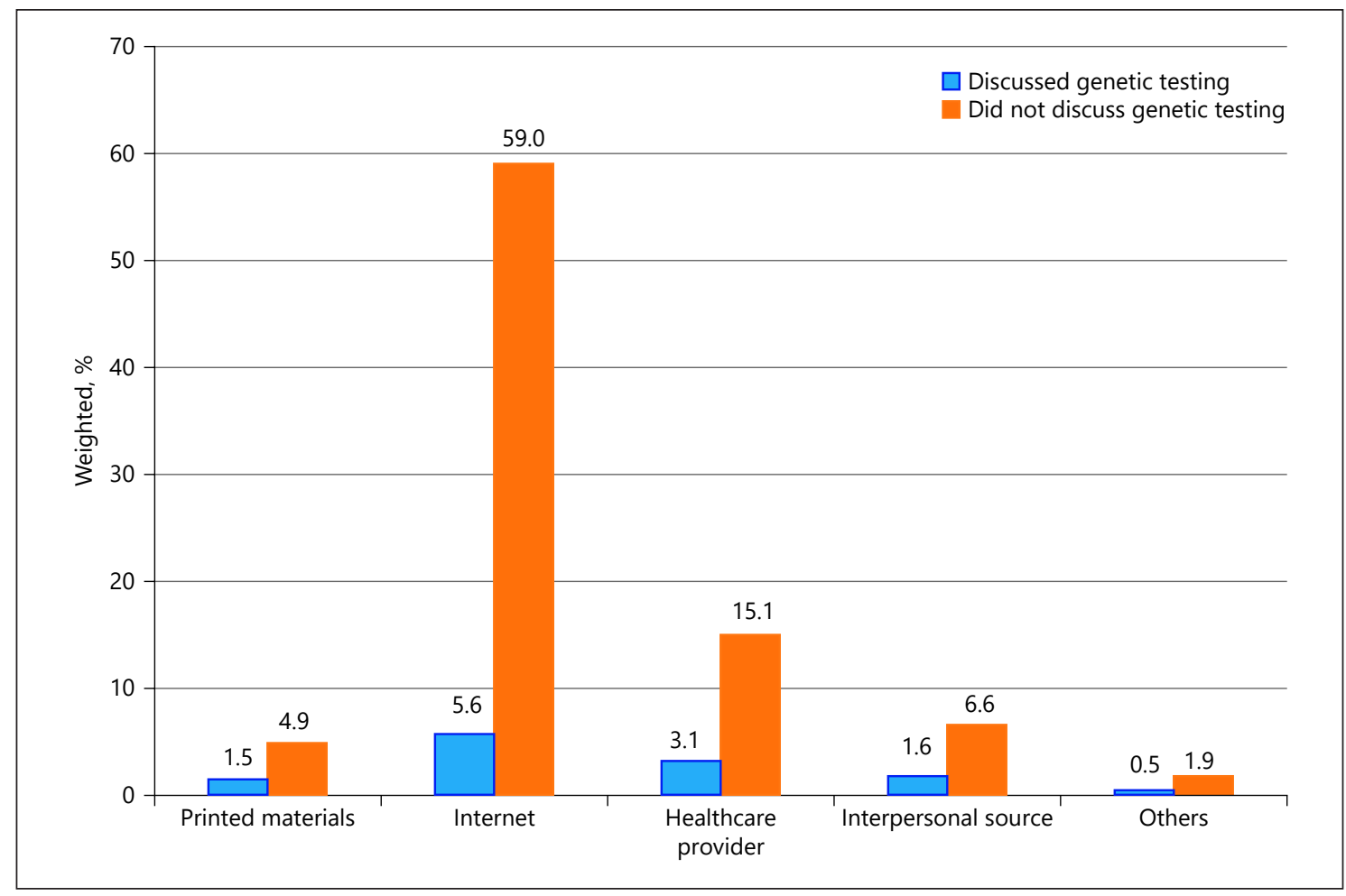

Fig. 2. Distribution of health information seeking sources between adults who did $(n=229)$ and did not $(n=$ $1,800)$ discuss cancer genetic testing with HCPs. HCP, healthcare provider. 


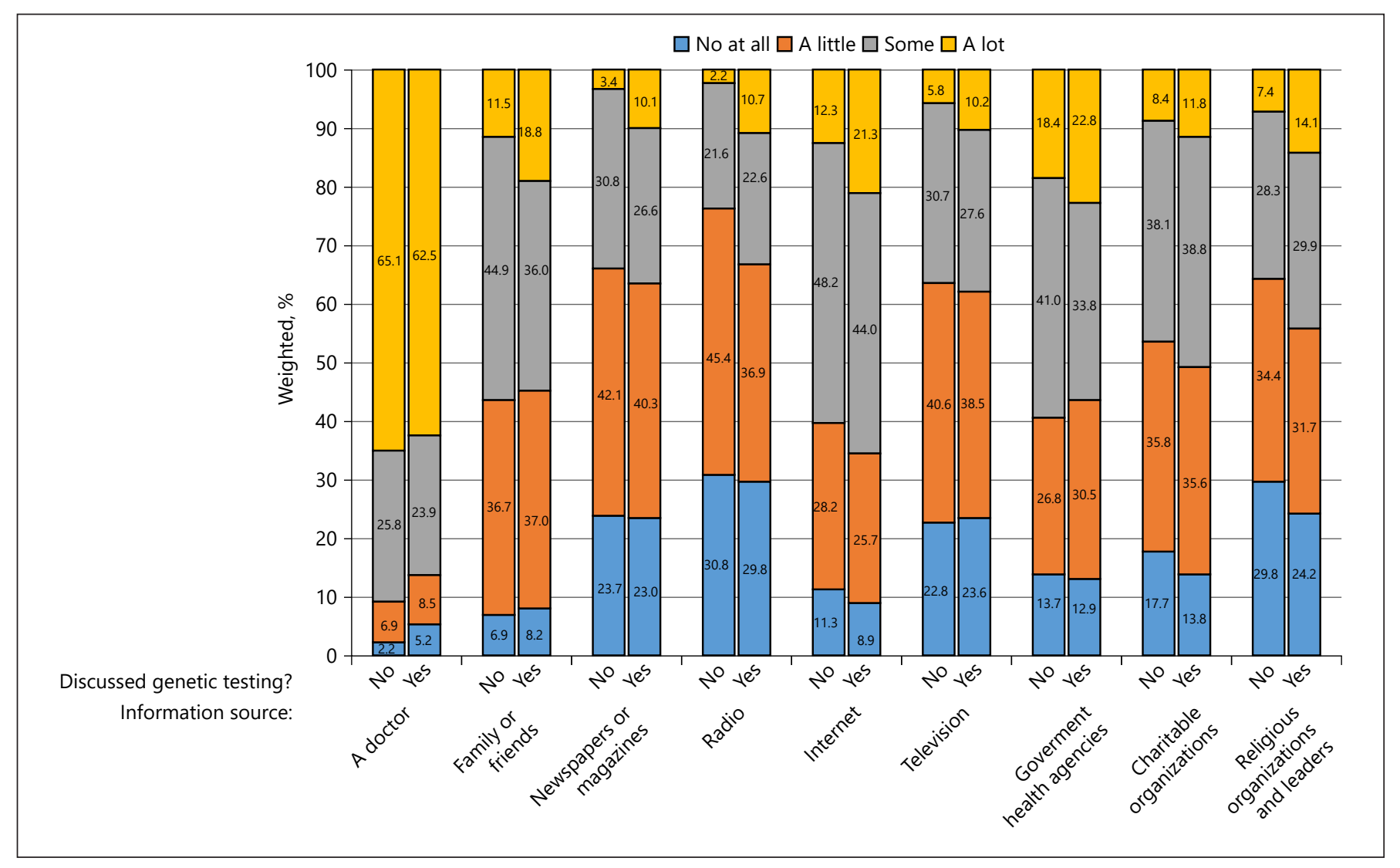

Fig. 3. Trusted information sources by discussion of cancer genetic testing among survey respondents.

Overall, 11\% ( $n=229)$ of respondents discussed genetic testing for cancer with a doctor or other health-care professional. Respondents who discussed were predominantly well-educated, married/living as married, and insured. Discussants were mostly non-Hispanic White (39.7\%), Hispanic (41.3\%), female (54.2\%), and urban resident (54.6\%). Mean age of discussants (42.1 years) was slightly lower than the mean age of nondiscussants (44.7 years). Most discussants (76.0\%) did not have any personal history of cancer but most had a family history of cancer (72.3\%) (Table 1). Distribution of psychosocial predictors stratified by discussion of genetics with HCP is presented in see online suppl. Table 1; see www. karger.com/doi/10.1159/000515465 for all online suppl. material.

Figure 1 shows the geographic distribution of survey responses by discussion of genetic testing. Such responses approximately represented the population distribution of Texas with most responses clustered around the 3 larger cities with a trend for fewer discussions reported in the historically underserved region of East Texas. There was no statistically significant difference in discussion of genetic testing with HCPs between respondents residing in urban and rural zip codes.

Health information seeking from any source was reported by $72.8 \%(1,461$ of 2,028$)$ of the study respondents. As shown in Figure 2, internet was the first source of information for most respondents (64.6\%), followed by HCPs (18.2\%) and interpersonal sources such as friends and family (8.2\%). Doctors were the most trusted source of information about health or medical topics (trusted "a lot" or "some" in the survey), followed by the internet and family or friends. Figure 3 shows the respondents' trust in various health information sources stratified by discussion of genetic testing.

\section{Predictors of Discussing Genetic Tests}

Psychological and sociodemographic predictor variables and covariates that were used in the multivariable model are shown in Table 2. Age influenced likelihood to have discussed genetic testing, with older participants being slightly less likely to discuss genetic testing with a pro- 
Table 2. Associations between sociodemographic and psychosocial predictors and discussion of genetic testing with HCPs among a Texas population-level sample $(n=2,029)$

\begin{tabular}{|c|c|c|c|}
\hline Predictor & OR & $95 \% \mathrm{CI}$ & $p$ value \\
\hline \multicolumn{4}{|l|}{ Sex } \\
\hline \multicolumn{4}{|l|}{ Female (ref) } \\
\hline \multicolumn{4}{|l|}{ Race/ethnicity } \\
\hline \multicolumn{4}{|l|}{ Non-Hispanic White (ref) } \\
\hline Non-Hispanic Black & 1.39 & $0.81-2.37$ & 0.236 \\
\hline Other & 1.30 & $0.68-2.50$ & 0.431 \\
\hline Age & 0.98 & $0.97-0.99$ & 0.011 \\
\hline \multicolumn{4}{|l|}{ Educational attainment } \\
\hline \multicolumn{4}{|l|}{ Up to 11 years (ref) } \\
\hline 12 years or completed high school & 1.68 & $0.80-3.53$ & 0.174 \\
\hline Divorced/widowed/separated/married/living as married & 1.19 & $0.79-1.80$ & 0.400 \\
\hline \multicolumn{4}{|l|}{ Household income } \\
\hline$<\$ 20 \mathrm{~K}$ & 0.89 & $0.52-1.53$ & 0.673 \\
\hline \multicolumn{4}{|l|}{$\$ 20$ to $<\$ 35 \mathrm{~K}$ (ref) } \\
\hline$\$ 35$ to $<\$ 75 \mathrm{~K}$ & 1.17 & $0.74-1.85$ & 0.501 \\
\hline$\$ 75 \mathrm{~K}+$ & 1.16 & $0.69-1.95$ & 0.585 \\
\hline Residence (rural vs. urban) & 1.11 & $0.74-1.66$ & 0.630 \\
\hline Health insurance (insured vs. uninsured) & 1.38 & $0.92-2.08$ & 0.123 \\
\hline \multicolumn{4}{|l|}{ Personal history of cancer } \\
\hline \multicolumn{4}{|l|}{ No cancer (ref) } \\
\hline Breast/ovarian/colon cancer & 11.57 & $5.34-25.03$ & $6.1 \times 10-10$ \\
\hline Other cancers & 3.18 & $1.69-5.97$ & $3.0 \times 10-4$ \\
\hline \multicolumn{4}{|l|}{$\begin{array}{l}\text { Compared to other people your age, how likely are you to get cancer in your lifetime? } \\
\text { Unlikely/very unlikely/neither unlikely or likely (ref) }\end{array}$} \\
\hline Likely/very likely & 0.96 & $0.65-1.41$ & 0.827 \\
\hline \multicolumn{4}{|l|}{ Cancer belief and preferences } \\
\hline \multicolumn{4}{|c|}{$\begin{array}{l}\text { How much do you agree or disagree with the following statement? I'd rather not know my chance of getting cancer } \\
\text { Strongly/somewhat disagree (ref) }\end{array}$} \\
\hline Strongly/somewhat agree & 0.82 & $0.57-1.19$ & 0.301 \\
\hline \multicolumn{4}{|c|}{$\begin{array}{l}\text { Which of the following do you believe has a significant effect on whether or not the average person develops cancer? } \\
\text { Not inherited predisposition such as cancer genes (ref) }\end{array}$} \\
\hline Inherited predisposition such as cancer genes and other factors & 0.54 & $0.36-0.79$ & $1.7 \times 10-3$ \\
\hline Inherited predisposition such as cancer genes only & 0.70 & $0.41-1.19$ & 0.190 \\
\hline \multicolumn{4}{|l|}{ How often do you feel depressed? } \\
\hline Daily/weekly/monthly (ref) & & & \\
\hline Never/a few times a year & 0.73 & $0.51-1.04$ & 0.081 \\
\hline It seems like everything causes cancer & & & \\
\hline Strongly/somewhat disagree (ref) & & & \\
\hline Strongly/somewhat agree & 0.92 & $0.63-1.34$ & 0.647 \\
\hline
\end{tabular}


Table 2 (continued)

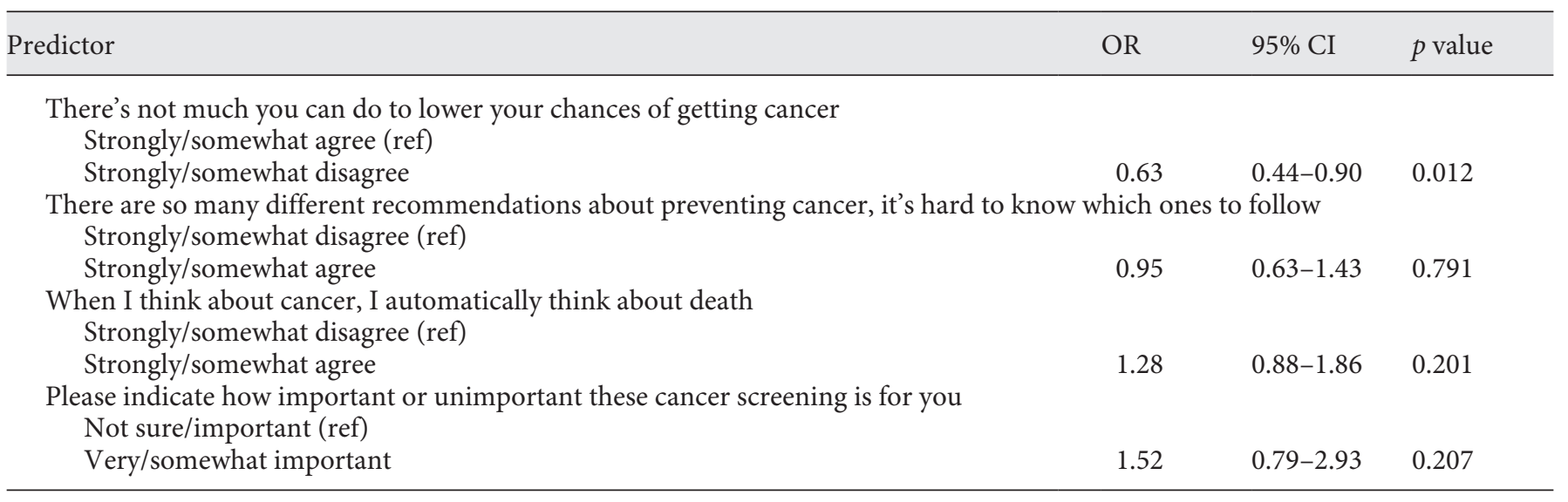

HCP, health-care provider. Statistically significant values are in bold.

vider $(\mathrm{OR}=0.98,95 \% \mathrm{CI}=0.97-0.99)$. Other sociodemographic characteristics such as sex, race/ethnicity, educational attainment, or income were not statistically significantly associated with discussion. Both clinical variables tested (i.e., personal and family history of cancer) were significant predictors of likelihood to discuss cancer genetic testing. Compared to participants without personal history of cancer, the odds of discussing genetic testing with HCPs was greatest for those with a personal history of prior breast, ovarian, or colorectal cancer $(\mathrm{OR}=11.57,95 \% \mathrm{CI}=5.34-25.03)$. Participants with personal history of other cancers were also more likely to discuss genetic testing with an HCP although the effect size was smaller $(\mathrm{OR}=3.18,95 \% \mathrm{CI}=1.69-5.97)$. Family history of cancer was also a significant predictor of discussing genetic testing compared to those who did not have or were unsure about family history of cancer $(\mathrm{OR}=1.66,95 \% \mathrm{CI}=1.13-2.45)$. Respondents who sought health information from any source were more likely to discuss cancer genetic testing with an HCP compared to those who did not seek health information $(\mathrm{OR}=1.73,95 \% \mathrm{CI}=1.12-2.66)$.

Of the psychological variables, believing that both inherited predisposition to cancer as well as other modifiable risk factors have a significant effect on cancer development was associated with a lower likelihood of discussing cancer genetic testing with an HCP compared to the belief that only other modifiable risk factors contribute to cancer $(\mathrm{OR}=0.54,95 \% \mathrm{CI}=0.36-0.79)$. Those who believed that it is possible to lower one's chances of getting cancer were less likely to discuss cancer genetic testing with a provider than to those who did not believe so

Discussions of Cancer Genetic Testing with HCPs
$(\mathrm{OR}=0.63,95 \% \mathrm{CI}=0.44-0.90)$. Notably, there was no difference in discussion of genetic testing with an HCP by participants' health insurance status.

\section{Discussion and Conclusion}

\section{Discussion}

Among the $>2,000$ Texas residents surveyed in 2018 in this study, $11 \%$ reported discussing the possibility of getting a genetic test for cancer risk with a doctor or other health professional across broad geographic regions of Texas. This rate is considerably higher than the $2.25 \%$ individuals who reported discussing genetic testing in the 2015 NHIS Cancer Control Supplement [9] and the 4.55\% discussion rate among women [27]. These differences can be attributed to several reasons. First, growing public awareness of genetic testing, with up to $57 \%$ of the US population reported to be aware of genetic testing in 2017 [28], has positively changed attitudes and interest in genetic testing, including cancer genetic testing $[29,30]$. Second, Texas is considerably more racially and ethnically diverse than the USA as a whole which may explain some proportion of this increase. Hispanics are reported as more likely to discuss genetic testing than non-Hispanic Whites [9], and they made up 35.6\% of our weighted study sample which is considerably higher than nationally representative samples such as NHIS [4]. Third, the landscape of clinical genetic testing has changed considerably since the last NHIS survey was conducted in 2015; eligibility criteria for clinical genetic testing has become more inclusive, and there is increased availability of ge- 
netic medical services. The sociodemographic characteristics associated with discussion of genetic testing were also somewhat different among Texas residents than the general US population. In contrast to prior research [28], younger participants in our study were more likely to discuss genetic testing with a provider, and there was no difference in discussion by sex in our sample. Educational attainment was not associated with discussion which is consistent with prior research where higher education, although associated with hearing about genetic testing, was not associated with increased discussion of genetic testing [9]. Some proportion of the study participants may have proactively initiated discussion of cancer genetic testing, while in other cases, providers may have initiated discussion as part of routine clinical cancer care for participants with a personal or family history of cancer. Indeed, our sample was enriched for individuals with personal (8.1\%) and family history of cancer (62.6\%), which were associated with higher likelihood of discussing genetic test options with a provider. Similar trends have also been observed in other nationally representative surveys [27]. Increased public awareness of genetic testing, as well as increasing adoption of evidence-based recommendations for genetic testing among HCPs, may explain the higher discussion rates reported here [31].

Younger participants and health information seekers were more likely to discuss cancer genetic testing with an HCP. Younger people are more likely to be aware of the latest genetic technology, including genetic testing, and be more skilled at health information seeking and thus may be more inclined to proactively discuss testing [31]. A hallmark of genetic cancer predisposition syndromes is their younger age of onset, knowledge of which may motivate younger people to discuss genetic testing with their providers. Although information alone does not guarantee healthy behaviors, acquiring adequate information may motivate individuals to make informed changes in their health practices, including discussing genetic testing with an HCP. In agreement with existing literature [5], participants in this study reported HCPs as the most trusted source of health information, whereas family and friends were not reported as major sources. Genetic testing is a relatively new phenomenon in medicine. Thus, it is reasonable that patients rely on HCPs for information about genetic testing since they are generally considered more trustworthy [32] and a credible source of useful medical information [33] compared to the internet, friends and family, or mass media sources. HCPs, particularly genetic counselors, provide highly tailored information that enables patients to acquire advice unique to their situation and preference for personalized material is known to be important to patients [34].

Interestingly, participants who believed that cancer prevention is possible were less likely to discuss cancer genetic testing with a provider. This may be explained by a lack of public awareness about genetic test result-driven cancer prevention and early detection measures. Most proactive discussions of genetic testing with HCPs likely stem from an expectation of health benefit. However, such discussions may not occur if patients are unaware of the existence of preventive measures for certain cancer predisposing conditions as well as cancer surveillance methods that can reduce morbidity and mortality [35]. This is also supported by the fact that respondents who believed that both nonmodifiable hereditary genetic cancer predispositions (perceived as deterministic [36]) and modifiable risk factors (such as obesity, tobacco, and alcohol) cause cancer were less likely to discuss genetic testing with a provider. For patients with significant family history of cancer, HCPs could also initiate discussion of genetic testing and primary care providers who are familiar with a patient's family history are uniquely positioned to play an active role in cancer prevention in this regard. Better awareness of genetics-informed cancer prevention methods in addition to awareness of genetic testing are needed for more widespread adoption of personalized genetic medicine.

It is unlikely that discussions of cancer genetic testing reported in this study included consumers seeking clinical confirmation of their DTC genetic test results as the first FDA approval of a DTC genetic cancer predisposition test was granted a day after this survey was completed, in March 5, 2018 [37]. Unwarranted discussions of genetic testing influenced by DTC testing and mass media are known to waste valuable provider time and have been mostly found to be a waste of health-care dollars [38]. This study, along with future iterations of this survey, is ideally positioned to enable before-after comparison of the impact that DTC cancer testing has on healthcare utilization trends in genomic medicine.

While our results have important implications, this study is not without its limitations. Self-reported survey data are vulnerable to recall bias, especially among those who underwent genetic testing. We cannot assess causality in this study given its cross-sectional design including whether information seeking and risk perception preceded or predicted discussion of genetic testing. This study utilized secondary data and future prospective studies that are purposefully designed to measure all constructs of the health behavior model (including self-efficacy and intention) to confirm the associations reported in this 
study are warranted. The survey did not collect sufficient personal and family history of cancer to evaluate eligibility for referral to genetic counseling (e.g., age at diagnosis and degree of relatedness with affected family member), or information on the specialties of participants' usual HCPs, and with whom they discussed genetic testing. In addition, the nonprobability sampling design may have enriched our sample for respondents with special interest in cancer prevention. Despite these limitations, the study has several strengths. This is a comprehensive and inclusive population-based study that provides populationrepresentative estimates of genetic testing discussion via the use of statistical weights. The study findings are derived from a survey conducted within the US health-care system and need to be replicated in non-US health-care systems to establish generalizability. Since this survey was completed only a few days before DTC cancer genetic testing became available, it provides important data regarding the clinical genetic testing landscape and can serve as comparison group for future studies.

\section{Practice Implications}

The results presented here show that discussion of cancer genetic testing with HCPs among Texas residents is higher than previously reported national rates. The finding that belief in the possibility of cancer prevention was related to lower discussion of genetic testing suggests that there may be limited public awareness of personalized cancer prevention methods that are guided by genetic test results. In order to optimize options for cancer prevention and early detection, genetic education initiatives to improve public awareness of personalized cancer prevention are needed to help at-risk individuals recognize their risk status and pursue appropriate genetic testing. The results of this study will help inform educational priorities in genetics needed to design materials suitable for the lay audience that can address use of genomic information in cancer prevention.

\section{Conclusion}

We report higher rate of discussion of cancer genetic testing with HCPs in our study population and identify individual situational factors (i.e., personal and family history of cancer) and psychological factors (i.e., belief that hereditary risk is important for cancer causation) that consistently influence these discussions. Our findings suggest a critical gap in public understanding of the genetic etiology of cancer and cancer-preventive health behaviors in Texas. Future studies to understand the public perceptions about genetic testing and cancer prevention in other countries and cultures are warranted. These data can directly inform global efforts in genomic medicine to identify patients who may benefit from genetic consultation [39]. Involvement of HCPs is critical for clinical genomic service delivery, and their involvement will be ever more important over the coming years with advances in scientific understanding of genomics, public awareness of clinical and DTC genetic testing, and use of genetics-informed cancer prevention methods.

\section{Statement of Ethics}

The research was conducted ethically in accordance with Declaration of Helsinki. A sample of adult Texas residents was identified among online research panelists retained by Qualtrics. The study participants have given their written consent and are managed by Qualtrics. MD Anderson's Institutional Review Board approved the study protocol and survey instrument before launching the study (PA16-0724).

\section{Conflict of Interest Statement}

The authors have no conflicts of interest to declare.

\section{Funding Sources}

This work was supported in part by the National Cancer Institute through Cancer Center Support Grant (CCSG) 5P30CA016672 (PI: P. Pisters), CCSG subproject 5697 (PI: S. Shete); the Assessment, Intervention, and Measurement Shared Resource through CCSG and the Duncan Family Institute for Cancer Prevention and Risk Assessment; a Cancer Prevention Fellowship supported by the Cancer Prevention and Research Institute of Texas grant award, RP170259 (to S.M.; PI: S. Chang and S. Shete); the Duncan Family Institute for Cancer Prevention and Risk Assessment (S.S.); and the Betty B. Marcus Chair in Cancer Prevention (S.S.).

\section{Author Contributions}

S.M. formulated the study concept and drafted the manuscript. R.Y. contributed to statistical analysis and interpretation of data. S.A.C., S.K.P.C., and S.S. were involved in critical revision of the manuscript for important intellectual content. S.S. was involved in formulating the study concept, design, and obtaining funding.

References

1 Garber JE, Offit K. Hereditary cancer predisposition syndromes. J Clin Oncol. 2005;23(2): 276-92.

2 Burke W, Culver J, Pinsky L, Hall S, Reynolds SE, Yasui Y, et al. Genetic assessment of breast cancer risk in primary care practice. Am J Med Genet A. 2009;149a(3):34956. 
3 Haga SB, Barry WT, Mills R, Ginsburg GS, Svetkey L, Sullivan J, et al. Public knowledge of and attitudes toward genetics and genetic testing. Genet Test Mol Biomarkers. 2013; 17(4):327-35

4 Stamp MH, Gordon OK, Childers CP, Childers KK. Painting a portrait: analysis of national health survey data for cancer genetic counseling. Cancer Med. 2019;8(3):1306-14.

5 Finney Rutten LJ, Agunwamba AA, Wilson P, Chawla N, Vieux S, Blanch-Hartigan D, et al. Cancer-related information seeking among cancer survivors: trends over a decade (20032013). J Cancer Educ. 2016;31(2):348-57.

6 Genetic/familial high risk assessment: breast and ovarian cancer. [2020 Jan 29]. Available from: www.ncen.org.

7 Genetic/familial high risk assessment: colorectal. [2020 Jan 29]. Available from: www. nccn.org.

8 Bellcross CA, Leadbetter S, Alford SH, Peipins LA. Prevalence and healthcare actions of women in a large health system with a family history meeting the 2005 USPSTF recommendation for BRCA genetic counseling referral. Cancer Epidemiol Biomarkers Prev. 2013;22(4):728-35.

9 Baer HJ, Brawarsky P, Murray MF, Haas JS. Familial risk of cancer and knowledge and use of genetic testing. J Gen Intern Med. 2010; 25(7):717-24.

10 Cigna MCP. Genetic testing for hereditary and multifactorial conditions. 2019. [cited 2020 Mar 13]. Available from: https://cignaforhcp.cigna.com/public/content/pdf/coveragePolicies/medical/mm_0052_coveragepositioncriteria_genetic_testing.pdf.

11 Reimbursement briefs: OIG, C.A.D. Cigna genetic testing policy takes effect. The Gray Sheet. Informa PLC; 2013.

12 Becker MH. The health belief model and sick role behavior. Health Educ Monogr. 1974; 2(4):409-19.

13 Reblin M, Kasting ML, Nam K, Scherr CL, Kim J, Thapa R, et al. Health beliefs associated with readiness for genetic counseling among high risk breast cancer survivors. Breast J. 2019;25(1):117-23.

14 Kasting ML, Conley CC, Hoogland AI, Scherr CL, Kim J, Thapa R, et al. A randomized controlled intervention to promote readiness to genetic counseling for breast cancer survivors. Psychooncology. 2019;28(5):980-8.

15 Waters EA, Wheeler C, Hamilton JG. How are information seeking, scanning, and processing related to beliefs about the roles of genetics and behavior in cancer causation? J Health Commun. 2016;21(Suppl 2):6-15.
16 Census, U.S. Quick facts Texas. 2020. [cited $2020 \mathrm{Jul}$ 30]. Available from: https://www. census.gov/quickfacts/TX.

17 Cunningham SA, Yu R, Shih T, Giordano S, McNeill LH, Rechis R, et al. Cancer-related risk perceptions and beliefs in Texas: findings from a 2018 population-level survey. Cancer Epidemiol Biomarkers Prev. 2019;28(3):48694.

182015 National Health Interview Survey. Survey Description. [cited 2020 Dec 22]. Available from: https://nhis.ipums.org/nhis/resources/srvydesc2015.pdf.

19 Nelson DE, Kreps GL, Hesse BW, Croyle RT, Willis G, Arora NK, et al. The Health Information National Trends Survey (HINTS): development, design, and dissemination. J Health Commun. 2004;9(5):443-4; discussion 81-4.

20 Nelson DE, Holtzman D, Bolen J, Stanwyck CA, Mack KA. Reliability and validity of measures from the Behavioral Risk Factor Surveillance System (BRFSS). Soz Praventivmed. 2001;46(Suppl 1):S3-42.

21 Case DO, Johnson JD, Andrews JE, Allard SL, Kelly KM. From two-step flow to the internet: the changing array of sources for genetics information seeking. J Am Soc Inf Sci. 2004; 55(8):660-9.

22 Morren M, Rijken M, Baanders AN, Bensing J. Perceived genetic knowledge, attitudes towards genetic testing, and the relationship between these among patients with a chronic disease. Patient Educ Couns. 2007;65(2):197204.

23 Roach AR, Lykins EL, Gochett CG, Brechting EH, Graue LO, Andrykowski MA. Differences in cancer information-seeking behavior, preferences, and awareness between cancer survivors and healthy controls: a national, population-based survey. J Cancer Educ. 2009;24(1):73-9.

24 Birkhäuer J, Gaab J, Kossowsky J, Hasler S, Krummenacher P, Werner C, et al. Trust in the health care professional and health outcome: a meta-analysis. PLoS One. 2017;12(2): e0170988.

25 Lee YJ, Boden-Albala B, Jia H, Wilcox A, Bakken $\mathrm{S}$. The association between online health information-seeking behaviors and health behaviors among hispanics in New York City: a community-based cross-sectional study. J Med Internet Res. 2015;17(11):e261.

26 Emanuel AS, Kiviniemi MT, Howell JL, Hay $\mathrm{JL}$, Waters EA, Orom $\mathrm{H}$, et al. Avoiding cancer risk information. Soc Sci Med. 2015;147: $113-20$.
27 Allen CG, Roberts M, Guan Y. Exploring predictors of genetic counseling and testing for hereditary breast and ovarian cancer: findings from the 2015 U.S. National Health Interview Survey. J Pers Med. 2019;9(2):26.

28 Krakow M, Ratcliff CL, Hesse BW, Greenberg-Worisek AJ. Assessing genetic literacy awareness and knowledge gaps in the US Population: results from the health information national trends survey. Public Health Genomics. 2017;20(6):343-8.

29 Henneman L, Vermeulen E, van El CG, Claassen L, Timmermans DR, Cornel MC. Public attitudes towards genetic testing revisited: comparing opinions between 2002 and 2010 . Eur J Hum Genet. 2013;21(8):793-9.

30 Etchegary H. Public attitudes toward genetic risk testing and its role in healthcare. Per Med. 2014;11(5):509-22.

31 Ashida S, Goodman M, Pandya C, Koehly LM, Lachance C, Stafford J, et al. Age differences in genetic knowledge, health literacy and causal beliefs for health conditions. Public Health Genomics. 2011;14(4-5):307-16.

32 Hesse BW, Nelson DE, Kreps GL, Croyle RT, Arora NK, Rimer BK, et al. Trust and sources of health information: the impact of the Internet and its implications for health care providers: findings from the first Health Information National Trends Survey. Arch Intern Med. 2005;165(22):2618-24.

33 Diaz JA, Griffith RA, Ng JJ, Reinert SE, Friedmann PD, Moulton AW. Patients' use of the Internet for medical information. J Gen Intern Med. 2002;17(3):180-5.

34 Ling BS, Klein WM, Dang Q. Relationship of communication and information measures to colorectal cancer screening utilization: results from HINTS. J Health Commun. 2006; 11(Suppl 1):181-90.

35 Ludwig KK, Neuner J, Butler A, Geurts JL, Kong AL. Risk reduction and survival benefit of prophylactic surgery in BRCA mutation carriers, a systematic review. Am J Surg. 2016; 212(4):660-9.

36 Khoury MJ, Thrasher JF, Burke W, Gettig EA, Fridinger F, Jackson R. Challenges in communicating genetics: a public health approach. Genet Med. 2000;2(3):198-202.

37 BRCA Basics. [2019 Sept 4]; Available from: https://www.23andme.com/brca/.

38 Tandy-Connor S, Guiltinan J, Krempely K, LaDuca H, Reineke P, Gutierrez S, et al. Falsepositive results released by direct-to-consumer genetic tests highlight the importance of clinical confirmation testing for appropriate patient care. Genet Med. 2018;20(12):151521.

39 Jacobs C, Patch C, Michie S. Communication about genetic testing with breast and ovarian cancer patients: a scoping review. Eur J Hum Genet. 2019;27(4):511-24. 